\title{
A Complexity Perspective on Presencing
}

\author{
OLEN GUNNLAUGSON \\ Université Laval (Canada)
}

This article outlines preliminary criteria for establishing a complexity perspective of presencing, a process-method of generative conversation that involves sensing, seeing into and apprehending complex emergent ways of knowing and inquiry within collective contexts of learning and inquiry. The article develops three central aims of complexity instruction as lenses through which to understand presencing as a basis for advancing the complexity project of engaging groups and teams through instructional processes that foster collective intelligence.

\section{Introduction}

To the extent that the broader aims of higher education remain bound by conventional instructional approaches involving the transmission and acquisition of pre-existing representational knowledge (Osberg \& Biesta, 2007; Osberg et al., 2008; Radford, 2007), as instructors we postpone or circumvent the exploration of complex emergent forms of knowledge making with our students. While discussions concerning principles of complexity and emergence have taken place in contexts of learning (Davis \& Sumara, 2007, 2006, 1997; Osberg, 2009), specific instructional practices that are capable of supporting and engaging the collective interior dimension of complex emergent processes of conversation for the purposes of fostering transformative forms of inquiry and a generative culture of learning within classroom collectives (Jorg, 2009) have not yet been addressed. This article examines presencing (Scharmer, 2007; Gunnlaugson, 2007, 2006) from a complexity perspective, focusing on discussions of key collective notions such as the "space of emergence" (Osberg \& Biesta, 2008), "enlarging the space of the possible" (Davis \& Phelps, 2004) and "teacher as the consciousness of the collective" (Davis \& Sumara, 2005). As this article will develop more in detail, further 
work is needed to identify instructional approaches for pursuing these normative and descriptive aims in classroom life. Leading into the conversation, I begin by drawing upon Scharmer's (2007) theory of presencing to inform the development of a process framework for apprehending emergent forms of knowledge within specific contexts of university instruction where generative learning is sought (i.e. leadership development courses and training, team-based learning, cohorts, many arts and social sciences courses, among others). Within each upcoming section, I inquire into how presencing sheds new interpretive light onto the above three aims of complexity instruction, exploring further considerations for how presencing can serve as a complex emergent process to inspire new collective modalities of learning and inquiry.

\section{Presencing a Space of Emergence}

The scientific notion of emergence, a key feature of complex systems, is helpful for conveying the creative dynamics involved within knowledge-creation processes of conversation. From the perspective of complexity theory, emergence arises from complex systems that create new properties from "autonomous unities coming together into larger, more powerful unities" (Davis \& Sumara, 2007). Osberg \& Biesta (2007a) differentiate between types of new properties with their distinction of weak and strong varieties of emergence. With the case of weak emergence, emergent properties are to varying extents constituted by what came before, yet contain something new (relative to what came before). For strong emergence, these new properties are not determined by previous conditions and in the context of what has come before are inconceivable and fundamentally new. The distinctions of strong and weak emergence are helpful in describing how both new emergent properties and emergent processes (Thompson, 2007) arise in complex systems. Where weak emergence is by definition characterized by conditioned and deterministic causes that give rise to novelty within a closed system, strong emergence is informed by the incoming of new non-determined causes that give rise to something completely new. Another way to think of the fruits of strong emergence is in terms of Goswami's (2001, p. 207-9) notion of fundamental creativity, which involves bringing about something new in a new context. Weak emergence can be compared to his (2001) notion of situational creativity, which involves bringing forth new combinations of old ideas in old contexts.

Scharmer (2007) depicts conversation as an emergent process characterized by four distinct yet interconnected fields of conversation, which is his term for the dynamic patterns of interaction that are evident in conversation (p. 271-272). Each field of conversation, for Scharmer, contains within it a characteristic intersubjective pattern that is informed by particular forms of engagement in listening and speaking within the group. In the framework below (Figure 1), Scharmer charts the natural evolution of distinct conversational fields, moving counter-clockwise from relatively closed and cautious conversation in the lower left-hand quadrant (i.e., downloading) through debate, dialogue and finally presencing. 


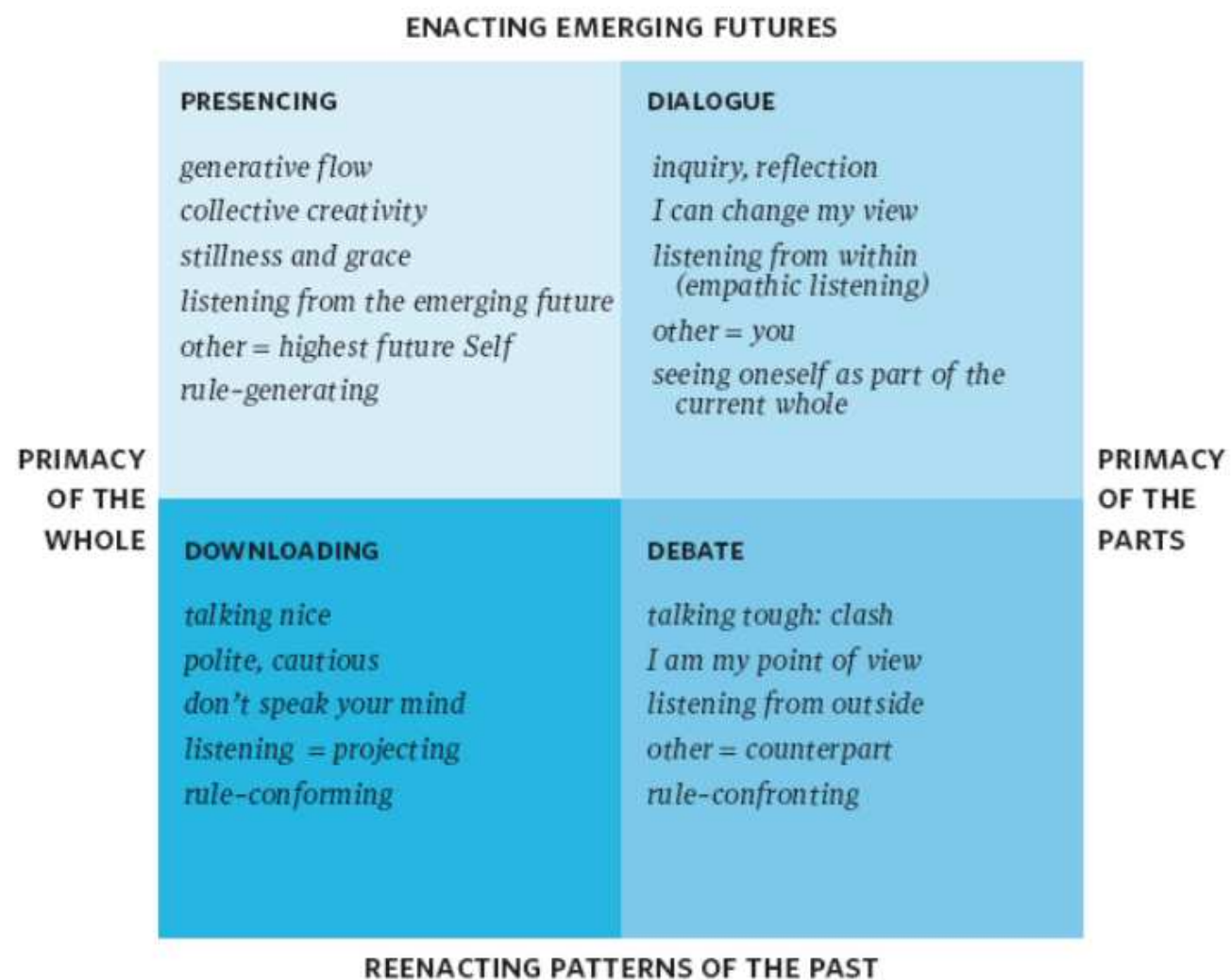

Figure 1: The Four Fields of Conversation (Scharmer, 2007, p.274)

As the fourth field of conversation, presencing involves a movement away from reflecting on the past or pre-existing representational knowledge, which is well known and underlies all conventional learning methodologies (Scharmer 2007, p. 7), towards learning together from the emerging future by collectively sensing into and intuiting not yet embodied or known possibilities. This shift in emphasis stands apart from transmission oriented pedagogical objectives that involve educating for the purposes of replicating past knowledge-objectives that are reinforced within the first two fields of downloading and debate and arguably, in many if not most fields of study within higher education contexts. Presencing is of particular interest here as it is constituted by and generated from a collective process of creative emergence. In complexity terms, the lower level social domain including the instructor and students gives rise to the higherlevel phenomenon of presencing as a field of conversation. To the extent that new meaning or knowledge arising in a presencing conversation is not deducible from the existing knowledge or understanding of the group (a characteristic tendency of previous fields of conversation) to return to Osberg \& Biesta's (2007a) previous distinction, we are participating in a process of strong emergence. Strong emergence has the potential to bring forth and open into new structures, ideas, forms of relationship and interaction, in turn becoming part of the history of individuals, groups, their learning processes and 
the institutional contexts of which they are a part. Unlike downloading or debate, which is characterized more by weak to insignificant emergence, the processes of strong emergence in presencing bring about the creation of more developed, complex and unpredictable pedagogical process-structures. Scharmer (2007) elaborates on presencing:

Time slowed down: space opened up. Several times ... I have encountered this shift to a deeper space of essential emergence. When it happens, time slows down and seems almost to stop, the atmosphere feels thicker, and my sense of space opens up, as if I were in a clearing or in a larger space... the boundary between me and my dialogue partners is now wide open, and we begin to operate from a common field (pp. 279-280).

Scharmer's brief phenomenological description of presencing suggests that the deeper relational experience of interconnectedness with the group is a vital condition for bringing about a collective co-enactment of the process of emergence. Scharmer elaborates, "presencing happens when our perception begins to connect to the source of our emerging future" (p.165), indicating that presencing offers the prospects of integrating and uniting the emerging observer with the emerging observed. Emergence in the context of presencing is not a systemically generated process or one devoid of the observer's participation within the greater field or system of conversation. Rather, the process involves attending more closely to one's own interiority and the shared field, inviting a more symbiotic process of thinking emergently with others. While new knowledge may arise from conversations that are not drawing from the process of presencing, presencing changes how we pay attention to the moment to moment unfolding of knowledge in conversation, making both ourselves and the knowledge we discover more prone to being emergent, open and creative rather than determined, closed and predictable (Scharmer, 2007).

From a complexivity perspective, a possible criticism of Scharmer's model of the four fields of conversation is that it utilizes "re-presentational" constructs, which are limited by pre-existing generalized patterns, to describe conversation as a process. Yet, upon closer inspection, it is important to note that Scharmer's framework draws from what Thompson and Varela (2001) in their account of emergent processes describe as, "local-to-global determination or "upward causation", as a result of which novel processes emerge that have their own features, lifetimes and domains of interaction" (p.416). Neither attempting to control or predict the emergent process or outcomes of conversation in advance, in using representational knowledge for emergentist purposes, Scharmer's model is more an example of what Johnson (2001) describes as the type of theory characteristic of the third phase of complexity theory, which involves the shift from the "analysis of emergence to the creation of emergence" (p.20). As such, one of the primary objectives of the four fields of conversation is to make the transition from the well established tradition within academic culture of re-enacting past forms of representational knowledge through discussion and debate to bringing about the conditions for the class to participate in and foster the emergence of new knowledge, ideas and perspectives in the conversational fields of dialogue and presencing. Davis \& Sumara (1997) have written briefly about conversation as a complex emergent phenomenon, drawing on Gadamer's notion of conversation as distinct from discussion 
and other forms of talk in conveying its importance as an interaction that cannot be predetermined:

The conversation is something more than the coordinated actions of autonomous agents -in a sense, it has us; we do not have it. Put differently, the conversation is not subject to predetermined goals, but unfolds within the reciprocal, codetermined actions of the persons involved" (1997, p. 5).

Fenwick (2003) also relates themes of complexity science to conversation as a "collective activity in which interaction enfolds the participants and moves beyond them" (p. 35). She goes on to convey how, from a systems view, a conversation is coinfluenced by the micro contexts of the participants, their relational space and patterns of interaction. In these descriptions, there is a confirmation of conversation serving as an example of complexity and emergence in the classroom, defined in part by the relational interactions between and among students, instructors and larger university, social and cultural systems. Though these perspectives bring attention to the general significance of conversation from a complexity perspective, to date there is no mention in the literature of how distinct forms of conversation might serve complexity objectives, in contrast to those forms that do not. From the perspective of Scharmer's (2007) four fields of conversation, conversation is viewed as an emergent creative system containing distinct micro contexts for working with the class as a collective field of generative learning. Each conversational field is shaped by characteristic field dynamics or patterns of engagement. While certain dynamics of listening and speaking arise in part out of the conditions enacted within the previous field, the content becomes increasingly indeterminable and new-particularly as one moves into the fourth conversational field of presencing. Within the complexity literature in education, the focus on conversation has been less as a critical micro context and more on the general interrelations of classroom learning events within complex systems (Fenwick, 2001) including the instructor, students, class subject, class environment, university culture and other dimensions of a larger, emergent process. @) As a point of contrast to how conversation has been framed within the complexity literature, Scharmer (2007, p.273) has taken a systems perspective on conversation one-step further by delineating particular corresponding systems within each field of conversation. His analysis is that field one (downloading) brings about an autistic system; in field two (debate), there is an adaptive system; in field three (dialogue), a self-reflective system and in field four (presencing), a generative system: 


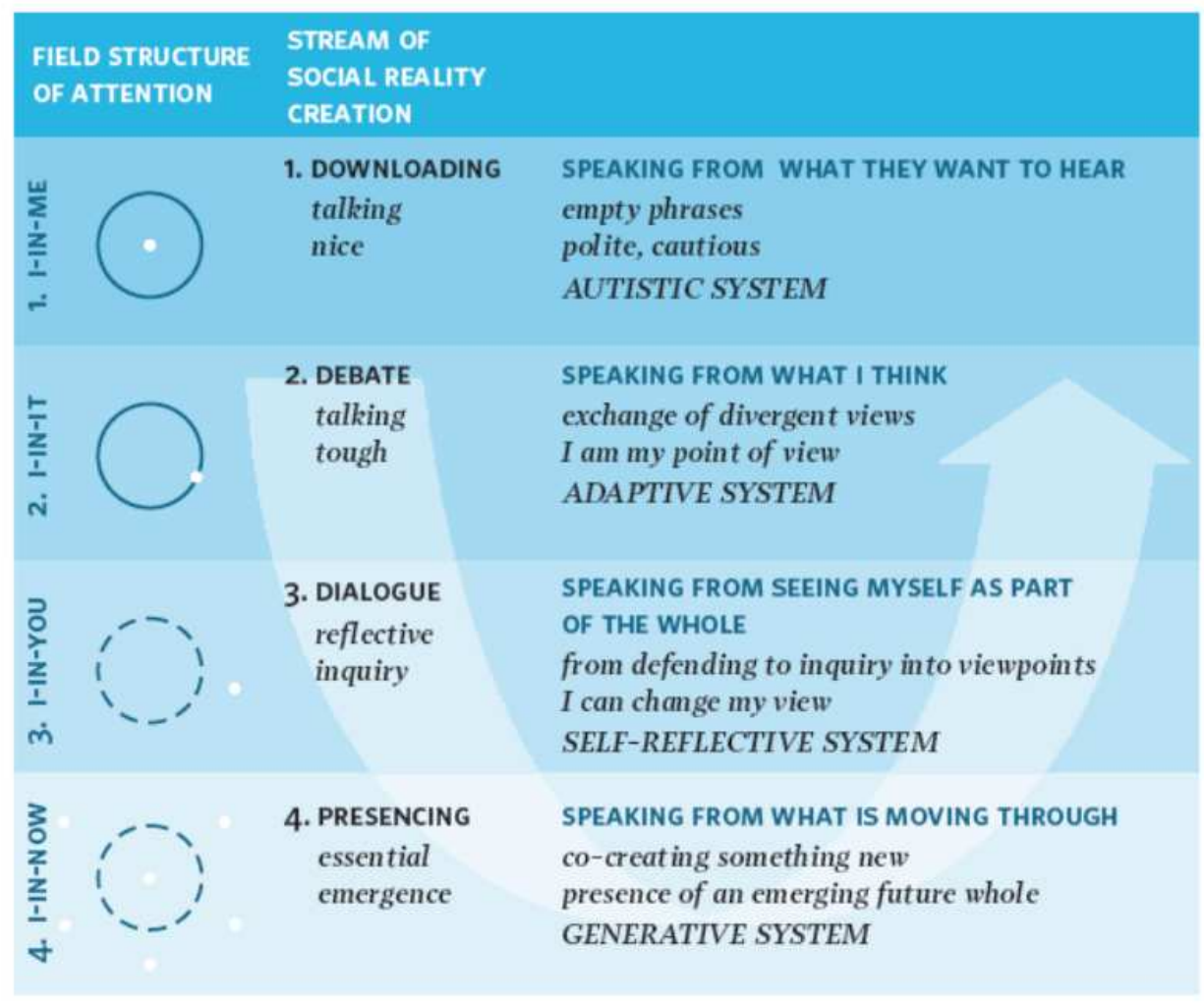

Figure 2: Four Fields of Conversation (2) (p.242)

When individuals in dialogue (field three) begin to realize they have been unconsciously participating in a system, awareness begins to dawn on the participants in terms of how they are participating in collectively enacting this system-not only of conversation, but the larger patterns of systems of culture, discourse, thought, etc (Scharmer, 2007). One might say that, in the field of dialogue, complexity awareness emerges. Here dialogue resembles Fenwick's and Davis \& Sumara's description of conversation, yet Scharmer's (2007) depiction of the meta-systemic awareness that develops from field three introduces the possibility of not only seeing one's participation within the systems one is apart of, but also moving into a new emergent system through a subtle shift in our self-identity, which tends to bring about an enlarged context for understanding a deeper sense of who we are. Peschl (2007) elaborates on the existential aspects of this shift that highlights an important aspect of presencing:

Profound change does not only happen in the cognitive domains, but touches a more fundamental level - an existential level that includes the person and his/her attitudes, values, habitus, etc. Whereas it is possible to "play games" on the cognitive/intellectual level (in the sense of trying out or simulating intellectual positions without being touched existentially by them), one can experience that there exists a level, where "intellectual games" are not possible any more. We are then confronted with a level going beyond the domain of cognitive or intellectual questions touching the self in the very center. (p.138) 
Experiences at the existential level in conversation can be helpful as catalysts for bringing about an emergent experience of our identity, which tends to recast the boundary lines of our individual consciousness and identity by re-centering our sense of self as a co-emergent participatory facet of the group-an experience that tends to interupt the individualistic, narcissistic tendencies of many of our students today (Astin \& Astin, 1999). Bohm's (1996) conception of dialogue as a method for exploring the subtle shared meaning that groups hold around a particular issue or subject is one example of how the field of dialogue, in contrast to debate or downloading, provides a context for exploring the interior ontological realms of meaning with individuals and collectives. In this way the latter fields of dialogue and presencing bring forth helpful shared conditions for exploring a particular subject with greater interpersonal discernment and depth. This positive de-centering of one's private sense of self is characteristic of the collective state of presencing as a participatory field of conversation, in which the potential for speaking and listening from a more fundamental existential and creative level becomes possible.

Returning to Figure 2, the shift from Scharmer's (2007) field of dialogue to the field of presencing involves enacting a generative system. As Scharmer points out, this tends to give rise to the experience of a connected, distributed, emerging sense of one's self that is thoroughly context-dependent and coupled to a surrounding emerging world. Though complexity theory does not raise specific existential or transpersonal challenges to such forms of group learning, presencing requires attending to, attuning with, amplifying and sensing into these dimensions of our experience as a basis for actualizing strong emergence and fundamental forms of creativity.

\section{Intuitions of the Incalculable}

Paradoxically, what we can know is potentially at once conditioned and unconditioned, known and unknown, with each conversation as knowledge-making event potentially offering us a deeper view into the unknown, unconditioned face of our existing knowledge. From the perspective of presencing, this paradoxical process is distilled from being present with, presencing into and looking from the hidden source or blindspot of our experience (Scharmer, 2007). Knowledge that emerges from the presencing process does not necessarily bring into question one's previous fund of knowledge or perspective. So, while presencing invites students into a distinct territory of learning, and though the emergent reality that unfolds may be incalculable from their previous experiences of conversation, a richer understanding of the possible arises. Such forms of emergence may not be predictable in a mathematical or scientific sense of the term. Yet, from the perspective of presencing, certain forms of emerging knowledge may be intuited and faintly sensed on the subtle level of our experience as a possibility beforehand by either the students or instructor. An example might be in the case of a team inquiring into a complex leadership problem from the level of deliberative discursive thinking, only to later on have aspects of the solution reveal itself through a particular revealing image that arises (and is not shared) from a team member when the group falls into an unintended silence. Then later on, upon the team's discovery of the 
solution, the team member may reflect upon this prior image, suggesting a kind of intuition at work.

As I have outlined (Gunnlaugson, 2006), intuition and related forms of knowing tend to become amplified in the field of presencing, in turn fostering an openness to experiencing a different sense of self and group identity, which introduces an important enlarging of the framework of the possible as described by Davis and Phelps (2004). For strong emergence of knowledge within a class, there cannot be a regrouping of preexisting knowledge (Morgan, 1923). According to complexity theory, knowledge that arises from strong emergence cannot be calculated beforehand (i.e., by reason or logic), yet intuition has not been satisfactorily accounted for in the complexity literature in education insofar as it works from a different form of measurement that generally confounds strict logical inference. Sorokin (1992) elaborates on intuition as follows:

Each source of knowledge-the senses, reason and intuition-affords a genuine cognition of the manifold reality. Intuition in its ordinary form as a momentary and direct grasp of a certain reality - the grasp distinct from sensory perception or logical reasoning-yields a knowledge of this aspect of reality. (p. 33)

Intuitive knowing can be challenging to convey and may in some contexts be more appropriately represented metaphorically by generative images, symbolism, stories and subtle forms of communication which are not easily accounted for by traditional assessment. Scharmer (2001) understands intuition as a form of self-transcending knowledge that requires a different kind of knowledge-creation environment:

Self-transcending knowledge relates to reality both from within and from outside. The locus of the denoted reality (outside the knower in the case of explicit knowledge and inside in the case of tacit-embodied knowledge) is both outside and within the knower. Or, as Nishida puts it, it is neither outside nor inside the knower (Nishida, 1990). From this point of view, knowledge emerges from a basho, a field or shared space. (p.142)

Unlike more mechanistic conceptions that identify the source of intuition as occurring from within individuals, for Scharmer intuition arises from a certain subtle contact with and articulation from this self-transcendent field or shared space that tends to be experienced paradoxically as both outside and inside the knower. In this sense, glimmers of emergent future knowledge may be partly present in advance and through intuitive forms of self-transcending knowing can be brought into fuller presentation of the present. For this to be so, we need to entertain the paradox of there being traces of an unimaginable future residing in the "not-yet embodied" (Scharmer, 1999) possibilities within the present that emerge out of absence into presence or the unknown into the known through individuals and groups. In describing the source of this intuitive knowledge as arising from the unknown, it is also important to acknowledge how we are constituted by the known images, narratives and discourses that we participate in. Presencing embraces this fundamental paradox by stimulating an unconditioned creative perception that aspires to be mindful of our past conditioning (in whatever form-whether from cultural, psychological, scientific or spiritual sources) and to be open and receptive to the non-systemic, non-conditioned, non-determined aspects of our 
experience, which are new. Presencing then involves opening a generative space or field of conversation where our identities, views, intuitions and inventions can be influenced by the unknown unconditioned source of our collective experience and the corresponding body of unimaginable knowledge that has not yet fully emerged or been lived into. In opening this generative space, presencing aspires to bring forth an order of experience that stands in contrast to the cultural studies critique that claims we are to varying degrees determined by the known images, narratives and discourses that shape our daily experience. In practice, it becomes necessary to develop a critical awareness of how these discourses shape our identities and self and world understanding. This is needed in order to sufficiently disentangle from them in order to more clearly apprehend the not-yet known images, narratives and discourses that have yet to emerge through us. This notion is implicit within David Bohm's (1980) theory of the implicate order, which posits a deeper hidden dimension of reality that is enfolded within the explicate order or dimensions of physical and psychological reality. According to Bohm, this underlying dimension of reality plays a role in shaping or informing what unfolds into our everyday experience of reality. In the context of strong emergence, presencing then might be thought of as a non-discursive process that attempts to glean insight from this underlying implicate order or field through intuition and other subtle forms of knowing. Such a notion would be viewed as a contradiction from the perspective of strong emergence, inasmuch as it is the unforeseeable outcomes of the processes of systemic emergence that is capable of bringing forth the unimaginable, not the emergent intuitions of our students in conversations oriented by presencing. Yet, if we place our conceptions of the unimaginable in the hands of unknowable systemic permutations, there is a risk that such a vision of what is unimaginable will no longer captivate our imaginations, insofar as the specific meanings, views and intelligence of the participants do not play a formative role in bringing us into a more profound experience of the unimaginable and incalculable. From the perspective of presencing, the present takes on a greater creative significance-a potential vehicle through which to encounter unknown facets of the strong processes of emergence. Presencing then draws in part upon intuition or a sense-making of a certain understanding of what is or what has yet to be, rather than relying solely on the structurally coupled parts of a complex emergent system to determine the process, measures or parameters of what is incalculable.

\section{Learning Presencing}

As each person contributes to the class conversation, in Scharmer's third field of dialogue, participants are committed to building on previous contributions. Often this involves drawing others into this occasioning through empathic forms of listening and reflective inquiry into shared meaning as a basis for experiencing a more authentic and personal engagement with others. As dialogue moves into the fourth field of presencing (Scharmer, 2007), students listen for and speak to what is emerging through them and the intersubjective field of conversation. In the presencing process, collective levels of cocreative engagement are emphasized, however the agency or voice of the individual learner is not neglected or surrendered insofar as the field of conversation is greatly 
contingent on shared resonance, shared interest and a shared process of creativity (Scharmer, 2007). While these shared processes alone do not ensure that personal subjectivities are fully acknowledged, they can become barometers that both instructor and students may utilize to ensure that underrepresented voices and views are sufficiently drawn upon and the individual is not deemphasized. Also, to offset traditional instructional practices that rest on problematic distributions of power, which can lead to privileging the instructor's knowledge, ways of learning, cultural practices and social relations, there is an ongoing need for encouraging participatory processes that lead to the co-unfolding of views between students and instructor within the class field.

As a way of teaching presencing, initially I have found it helpful to introduce, facilitate and model presencing forms of conversation as distinct from dialogue and related types of conversation. However, with practice and guidance over the term, students are encouraged to rely less on the instructor as a coordinating agent and more on their own discernment in relation to the group as a basis for a process or fieldcentered classroom. From the perspective of the instructor as facilitator, control of the emergent process is released so that other forms of collective intelligence can arise from the class field as a collective learner. The locus of authority then is intentionally distributed from the instructor to include the students and field of conversation. Preceding the connected quality of self and knowing that emerges when students are presencing together is a way of listening and being that is attuned initially to their own interior promptings, but then gradually more and more to the subtle depths of what wants to emerge from the group field of conversation.

Learning presencing generally involves introducing a dynamic, nonlinear and group-organized process that is well matched with course objectives and instructional approaches that value complex emergent forms of knowing and knowledge-creation. Whether for developing creative responses to complex leadership problems, addressing future oriented learning matters within a given field of specialization, or fostering student's capacity for engaging in collective creative processes that build on established self and world knowledge, presencing can be adapted to assist the exploration of coursespecific content within higher education classrooms where the aforementioned and related purposes play a central role.

From a complex emergence perspective, the presencing process needs to embrace the "mistakes" and "unexpected events" of the group process as important aspects of an emergent curriculum. Moving away from a "spatial" representation of knowledge (Osberg et al., 2008) that is evaluated by its degree of objective correspondence with reality, a more "temporal" epistemology is needed-one that involves "finding more and more complex and creative ways of interacting with our reality" (p. 215). This is not to say that a temporal representation replaces a spatial one, more that our instructional commitments shift from prioritizing pre-existing knowledge to valuing emergent knowledge. Entering into the space of liminality offers a way to proceed with a temporal epistemological approach in our classrooms, as conveyed by Linds (2004) 
The word limnos, meaning threshold defines that space between certainty and uncertainty, between what was and what will be. The process contains doubt as well as certainty, and is simultaneously orderly and disorderly, and both rational and intuitive. Liminality offers a space in which to hold things in a tentative way. It provides an opening where we might move beyond singular truths and examine multiple possibilities. In such encounters we can begin to recognize the limitations of our own perceptions of "what life is like." Maturana and Varela (1992) call this recognition the social imperative for a human-centered ethic. Whenever we find ourselves "holding tightly to certainty," thinking we know the "best right way," we can interrupt this certitude and invite ourselves to step into "another domain where coexistence takes place" (Maturana and Varela, 1992). (p. 6)

Liminality provides an expanded temporal context for the emergent process of presencing to unfold. Presencing opens us into liminal spaces through conversation, sensitizing us to an awareness of how knowledge creation processes take place in time. Preoccupations with past knowledge tend to foster a closure of our perceptual faculties and risk unwittingly adhering to McLuhan's adage of seeing the world through a rear view mirror, driving into the future in reverse. To connect with what is emerging, we need to find ourselves in time and come to grips with this deep conditioning of orienting through past conceptions and pre-conceptions. Creative horizons then open through liminal experiences that interrupt the traditional academic adherence to a spatial representation of the world and one's self. Liminality requires a willingness to abide in not knowing and to priming other faculties of knowing as a way of grappling with the existential implications of not knowing and the encumbered feelings of groundlessness this tends to invoke for students well accustomed to leading from the known and the past. Attention held well in the dimly lit regions of liminality primes our own and the group's awareness for an unexpected discovery - that is a presence-led form of seeing that is capable of comprehending the deeper challenges that might otherwise discourage us from opening up into what we have not yet imagined.

\section{Deepening the Space of the Possible}

Reflecting further on the complexity project of enlarging the space of the possible, Osberg (2009, p. vi) points out, "If we can already imagine what is possible, achieving such is no longer enlarging the space of the possible, for it already exists in our minds." Osberg draws on Derrida's notion of the impossible (i.e., what currently cannot be conceived as possible) as a basis for augmenting what is possible. She also suggests that experimenting with principles of complexity thinking, which does not rely on a linear logic of cause and effect, is better equipped to expand the horizons of the possible through an emergent process, pointing out that, "when emergence takes place, we enter the space of the impossible or incalculable" (p. vii). Invoking a dynamic interaction in the classroom, in contrast to linear thinking, informed by either weak or strong emergence by definition then allows us access Osberg's notion of the impossible (p. vi). Building on Osberg's point, consider a complementary approach that draws upon but is 
not exclusively determined by complexity processes in augmenting the space of the possible? For this to be so, we need a basis of assessment for orienting ourselves towards the unknown with our students. In brief, the ideal of the impossible needs to be augmented to include not only the measures of structurally coupled systems and emergentist logic but also interior measures of meaning and value drawing on the personhood of participants in the conversation and the collective.

From a presencing standpoint, attending to the emergent meaning of the individual and collective is an essential barometer in determining whether or not such impossible forms of emergent knowledge are of value. Instructing and leading from presencing then involves uncovering a basis for augmenting the space of the possible by exploring grounds for a meaningful impossible by attuning to the life worlds of participants and the emerging dynamics of the conversation. III.1 Being with What Is

When conversations are occasioned by presencing, the group field becomes the representative context or signifier where our understanding and shared meaning are renewed and evolving. Complexity approaches to learning acknowledge the unconditioned aspect of the present insofar as the present is always a new present, to the extent that each moment has an emergent nature. Nevertheless, while this is true in principle, presencing in conversation engages with the present in such a fashion that this newness can be understood ontologically through presence-that is, through a fundamental shared experience of who we are. Orienting from presence helps us become aware of our conditioned modes and filters of seeing and being in the present. With practice, this helps us draw from the unknown and unforeseen side of what is, thereby making way for the arrival of new discoveries. Under such conditions, conversations become a collective lens through which emergent knowledge can be brought into focus.

Paradoxically, presencing offers a different emphasis on being with what is as a basis for discovering a deeper creative dimension of what might be brought forth. Presencing involves amplifying our experience of the present so it can serve a generative function or become a generative context out of which new discoveries can emerge. Contrary to Davis et al.'s (2003) claim that the emergence does not emphasize what is, but what might be brought forth (p. 228), in the context of presencing the commitment to being present with what is becomes an essential means for bringing forth emergence within the presencing process. What is, in the context of presencing, is a referent and invitation to being mindful and encountering our experience of a specific subject of inquiry more fully in the present-including our assumptions, mental models and ways of understanding. When we experience the deeper dimensions of what is, there is a distinctive quality of awareness and sensing into a particular subject, situation or conversational inquiry. Being with what is then becomes an invitation to engage one's presence with what is emerging and what wants to emerge-affording ways of seeing and sensing into the very process of emergence itself in the context of conversation or other creative pursuits. Being with what is offers a point of contact with the emergent nature of reality as Tenzin Palmo (2000) elaborates on the nature of self-deception: 
There's tremendous resistance in the mind to being in the present, to just being with what is, rather than with all our fantasies and projections about how we want life to be. Just seeing life as it is, without any of our commentaries is very hard. For example, when I look at an object, I immediately start thinking of others I've seen which were similar, of whether I like the shape or don't like the shape, of whether the workmanship is good or not good, of how I might have wanted one which was somewhat different. This goes on infinitely - elaborating, elaborating, and elaborating until we don't see the object at all any more. (p. 4)

By orienting in part from what is, we are attending to the deeper source of knowing from within our experience, affording consciousness and our subjectivity a more direct role in augmenting the horizons of possibility. Learning to be with what is as a gateway to sensing into what will be provides a contrasting contemplative path to opening up spaces of the not-yet imaginable. Though the present contains the already imagined and existing possible, for Scharmer (2007) the present offers a window into the emerging future. However, if our individual/shared interior consciousness is not participating in the process, there is a risk that participants are merely orienting their attention to the exploration of the space of the possible without connecting with their deeper sources of wisdom and meaning that otherwise makes such pursuits worthwhile.

\section{Instructor as the Consciousness of the Classroom Collective}

Davis and Sumara (2007, 2006, 2005) as well as Davis and Simmt $(2006,2003)$ offer suggestions to guide the conditions for emergence. I will revisit the first three of the five necessary conditions from the context of presencing as initially outlined by Davis and Simmt (2003).

First, the notion of internal diversity suggests that instructors can benefit from attending to occasions where students' "various interests, capacities, experiences, milieus and personalities" (Bowsfield, 2004, p.148) as well as historically and culturally underrepresented perspectives can be woven into the conversation-becoming a part of the group's source of collective intelligence. Internal diversity offers a helpful principle for students to explore moving into a fuller expression and integration of who they are individually in relation to the subject they are presencing into. By encouraging students to attend to their socio-cultural location and particular history in terms of social class, gender, age, sexual identity, racial heritage among other representations of diversity, the class conversation is less prone to being directed from the dominant status quo of the particular culture one may be instrucing from, as well as our entrenched habits of thought, and self and world view. Secondly, internal redundancy is the common ground or shared elements of relationship, language, experiences, values and worldviews that enable the emergence of a group culture. Internal redundancy ensures that diversity is held in a larger shared context that fosters the conditions for students to feel safe to exchange and develop new knowledge and share from the diverse emergent meanings that arise. Within the field of presencing, participants explore the diversity of views, responses and perspectives with the objective of uncovering shared resonance, which offers a phenomenological barometer for shared meaning and shared ground. When 
students resonate with one another or a point that has been made by a fellow student, this raises collective attention and interest, which in turn may provoke resistance or disagreement by other students. Thirdly, decentralized control involves dispersing control through a shared responsibility for learning-centered participation in the processes of classroom emergence. In the context of presencing, decentralized control is a natural outcome of shifting one's locus of identity from an individual skinencapsulated ego to a more spacious presence alongside others unfolding a greater participatory process of emergence.

Drawing attention to the importance of attending to the interplay of these diverse elements, Davis and Sumara recast the instructor as the consciousness of the collectivein terms of looking for ways to broaden the canvas of possibilities for learning in collective contexts through the application of complexity principles. To the extent that presencing relies on the individual and collective interior processes of students and the group, the process is more concerned with deepening student's experience of learning within the collective through the field of conversation. Davis \& Sumara's (2005) description of the class "as a learner-not a collection of learners, but a collective learner-with a coherence and evolving identity all of its own" becomes possible through mastering the complexity principles discussed above.

In terms of the distinctions of Scharmer's (2007) four fields of conversation, the class field of presencing becomes the chief context in which Davis \& Sumara's notion of the collective learner emerges. For the presencing instructor committed to bringing forth the collective learner, a degree of mastery in co-creating the conditions for presencing are needed in order to avoid a reductionistic engagement with our students, in turn relegating the dynamic emerging gestalt of the collective to a fanciful impractical possibility.

\section{Coming into Presence with Others}

To go into the gap, to "descend into that alien territory," entails both a risk and an opportunity. The risk is clear: The space of enunciation is in a very fundamental and practical sense unpredictable. Yet it is at the same time the space in which speaking becomes possible, it is the space, in other word, where people-individual singular beings - can reveal who they are, can come "into presence" (Biesta, 2004, pp. 21-22).

Vanderstraeten \& Biesta (2001) locate the educational situation in the in-between-space of conversation, orienting from the tension points of difference between possible worlds and actual worlds as well as their unique subjectivity. In their conversation, they arrive at the notion that the in-between-space of conversational interaction is that which educates (p.17). By giving primacy to what happens between students, for Biesta (1999) the process of communication and the intersubjective context take precedence for the educational situation and the experience of coming into presence. Whether our students or the world, according to Osberg \& Biesta (2007a) both are continually in a state of coming into presence. This has important implications for our instructional practice, insofar as coming into presence, like presencing in the context of conversation, takes 
place in an intersubjective context-a world shared by others who are both like and unlike us. What, then, is required to shift our orientation towards coming-into-presence, presencing and other methods that facilitate complex emergence without socializing our students into a prescribed way of being or the replication of a particular socio-cultural learning ideal, regardless of how progressive, emergent or desirable? I ask this question from the perspective of presencing, as it requires some structured guidance and practice for orienting one's attention with others in conversation that is distinct from the accustomed representational way of communicating in conventional forms of discussion and debate. By encouraging different approaches to presencing that facilitate the coenactment of deeper conversational fields of co-emergence, students are free to explore their own approach, while simultaneously recognizing that a certain degree of social patterning in group work or teams is not necessarily undesirable. Especially as presencing tends to soften the influence of our social roles, inviting situations of learning where the field of conversation becomes the instructor, the student becomes the instructor with the instructor directing her attention towards primarily serving her students and the shared field of inquiry as a catalyst for such occasionings.

For the purposes of imparting methods of curriculum and instruction, an appropriate modality of training is required, particularly one that is sensitive to Osberg \& Biesta's (2008) critique of the extremes of unguided learning and enculturation. At some level we need to introduce, provide guidance in and represent what is involved with presencing. A complex emergent process is thus needed to depart from the logic of either unguided learning, which fails to enact appropriate boundaries and conditions for presencing, and enculturation, which fails to honor the emergent process of shared and individual meanings and subjectivities that play a central role in presencing. Such a learning process must come to terms with the paradox that forms of conversation are possible that do not "direct the subjectivity of others" (Osberg \& Biesta, 2008), but rather nurture a collective individualism which facilitates the emergence and transformation of students in ways that leave open the inquiry of what it means to be a human subject in our time and what it means to instruct from a collective complex emergent process of conversation that is committed to fostering the complexity ideals explored above.

\section{Closing Thoughts}

This article has explored how Scharmer's account of presencing as a field of conversation contributes to the project of developing a process framework for engaging complex emergent processes in group- or team-based contexts of generative learning. I have engaged with and, in some cases, re-interpreted certain key notions within the complexity education literature from the perspective of presencing for the sake of supporting non-deterministic fields of generative classroom conversation that can bring new insights into the role of the class field as a collective vehicle for transformative forms of self and knowledge-creation processes. In raising a challenge to complexity educational practices and aims, presencing asks that we explore the depth dimensions of being that co-arise within interwoven classroom and team-based group systems- 
orientating in part from what emergence feels like from ontological contexts of being from within the individual and collective as a basis for exploring complexity dynamics. Additionally, an integration and further inquiry into the existential and intuitive dimensions of collective complexivist learning processes are needed. By examining the significance of conversational fields from a complexity perspective throughout this article, my intention has been to identify a process framework through which complex emergent phenomena of learning can be engaged collectively. By bringing a complexity perspective to conversation and the underlying patterns that inform presencing, this article offers a lens to engage with and bring about transformation of the complex emergent processes and outcomes that characterize collective approaches to learning.

\section{References}

Araujo, F. (2008). Crossroad: The liminal state of light and dark-Solo show in Miami. Retrieved April 14, 2009, from॰ http://www.artistshowdown.com/fernando-ferreira-de-araujo-solo-show-in-miami

Astin, A. \& Astin, H. (1999). Meaning and spirituality in the lives of college faculty: a study॰ of value, authenticity and stress. Higher Education Research Institute Monograph (Los Angeles, University of California). Atkinson, T., \& Claxton, G. (2000). The intuitive practitioner: On the value of not always knowing what one is doing. Buckingham: Open University Press.

Biesta, G. (2004). Mind the gap!: Communication and the educational relation. In C. Bingham \& A. Sidorkin (Eds.), No education without relation (pp. 11-22). New York: Peter Lang Publishers.

Biesta, G. (1999). Radical intersubjectivity. Reflections on the "different" foundation of education. Studies in Philosophy and Education, 18(4), 203-220.

Bohm, D. (1980). Wholeness and the implicate order. London: Routledge.

Bohm, D. (1996). On dialogue. London \& New York: Routledge Publishers.

Bowsfield, S. (2004). Complexity in the English language arts classroom: Prompting the collective. Proceedings of the 2004 Complexity Science and Educational Research Conference, Sep 30-Oct 3. Chaffey's Locks, Canada.

Burris, E.D. (2005). Structure determinism and psychoanalytic theory: A wedding of fractals. Paper presented at the Complexity Science and Educational Research Conference, Nov 20-22. Robert, LA.

Davis, B., \& Phelps, R. (2004). Complicity: An introduction and a welcome. Complicity: An International Journal of Complexity and Education, 1(1), 1-7.

Davis, B., \& Simmt, E. (2006). Mathematics-for-teaching: An ongoing investigation of the mathematics that teachers (need to) know. Educational Studies in @) Mathematics, 61(3), 293-319.

Davis, B., \& Simmt, E. (2003). Understanding learning Systems: Mathematics education and complexity science. Journal for Research in Mathematics@ Education, (34), 137-177.

Davis, B., \& Sumara, D. (2007). Complexity science and education: Reconceptualizing the teacher's role in learning. Interchange, 38(1), 53-67.

Davis, B., \& Sumara, D. (2006). Complexity and education: Inquiries into learning, teaching, and research. Mahwah, NJ: Lawrence Erlbaum.

Davis, B., \& Sumara, D. (2005). Challenging images of knowing: Complexity science and educational research. International Journal of Qualitative Studies in () Education, 18(3), 305-321.

Davis, B., \& Sumara, D. (1997). Cognition, complexity, and teacher education. Harvard Educational Review, 67(1), 105-125.

Davis, B., Sumara, D., \& Simmt, E. (2003). Complexity and collectivity: On the emergence of a few ideas. In Davis (Ed.), Proceedings: Proceedings of the® first conference on complexity science and educational research, Oct 16-18. Edmonton, Alberta: University of Alberta. 
Fenwick, T. (2003). Learning through experience: Troubling assumptions and intersecting questions. Florida: Krieger.

Fenwick, T. (2001). Tides of change: New themes and questions in workplace learning. In T. Fenwick (Ed.), Sociocultural perspectives on learning through work (pp. 3-17). San Francisco: Wiley/JosseyBass.

Goswami, A. (2001). The physicists' view of nature: Part 2: The quantum revolution. New York: Kluwer Academic/Plenum Publishers.

Gunnlaugson, O. (2007). Revisioning possibilities for how groups learn together: Venturing an AQAL model of generative dialogue. Integral Review, (3)1, 44- 58.

Gunnlaugson, O. (2006). Exploring generative dialogue as a transformative learning practice within adult \& higher education settings. Journal of Adult and Continuing Education, 12(1), 1-19.

Karpiak, I. E. (2003). The listening heart: Tuning in for transformative learning, Proceedings of the Invitational Conference on Complexity Science and Educational Research, Oct 16-18. University of Alberta, Edmonton, Alberta. Johnson, S. (2001). Emergence: The connected lives of ants, brains, cities, and software.๑ New York: Scribner.

Jorg, T. (2009). Thinking in complexity about learning and education: A programmatic view. Complicity: An International Journal of Complexity and Education, 6(1), 1-22.

Linds, W. (2004). Stopping in-between: (Inter)playing moments of a theatre workshop. Educational Insights, 9(1). Available: http://www.ccfi.educ.ubc.ca/publication/insights/v09n01/articles/linds.html

Maturana, H., \& Varela, F. (1992). The tree of knowledge: The biological roots of human understanding. (Revised edition) Boston, MA: Shambhala.

Morgan, C.L. (1923). Emergent evolution. London: Williams and Norgate.

Osberg, D. (2009). "Enlarging the space of the possible" around what it means to educate and be educated. Complicity: An International Journal of Complexity and Education, 6(1), iii-x.

Osberg, D., \& Biesta, G. (2008). The emergent curriculum: navigating a complex course between unguided learning and planed enculturation. Journal of Curriculum Studies, 40(3), 313 - 328.

Osberg, D.C., Biesta, G.J.J., \& Cilliers, P. (2008). From representation to emergence: Complexity's challenge to the epistemology of schooling. Educational Philosophy and Theory, 40(1), 213-227.

Osberg, D., \& Biesta, G. (2007a). Beyond presence: Epistemological and pedagogical implications of 'strong' emergence. Interchange, (38)1, 31-57.

Osberg, D., \& Biesta, G. (2007b). Rethinking schooling through the "logic" of emergence: Some thoughts on planned enculturation and educational responsibility. In Geyer, R., \& Bogg, J. (Eds.) Complexity, Science and Society (pp. 35-38). Oxford, New York: Radcliffe Publishing Ltd.

Palmo, T. (2000). Gatsal teachings: Patience and effort. Retrieved February 15th, 2009: http://tenzinpalmo.com/index.php?option=com content\&task=view\&id=46\&It emid=36

Peschl, M. F. (2007). Triple-loop learning as foundation for profound change, individual cultivation, and radical innovation. Construction processes beyond scientific and rational knowledge. Constructivist Foundations, 2(2-3), 136-145.

Radford, M. (2007). Prediction, control and the challenge to complexity. Oxford Review of Education, 34(5), 505-520.

Scharmer, C. O. (2007). Theory U: Leading from the future as it emerges. SoL Press, Cambridge, MA.

Scharmer, C. O. (2001). Self-transcending knowledge: Sensing and organizing around emerging opportunities. Journal of Knowledge Management, 5(2), 137-150.Scharmer, C. O. (1999). Organizing around not-yet-embodied knowledge. In: Krogh, G., Nonaka, I., \& Nishiguchi, T. (Eds.), Knowledge Creation: A New Source of Value, (pp. 36-60). New York: Macmillan.

Sorokin, P. A. (1992). The crisis of our age. Chatam, NY: Oneworld Publications.

Thompson, E. (2007). Mind in life: Biology, phenomenology, and the sciences of mind. Cambridge, MA: Harvard University Press. 
Ulmer, G.L. (1985). Applied grammatology: Post(e)-pedagogy from Jacques Derrida to Joseph Beuys. Baltimore: Johns Hopkins University Press.

Vanderstraeten, R., \& Biesta, G. (2001). How is education possible? Preliminary investigations for a theory of education. Educational Philosophy and Theory, 33(1), 7-21.

\section{About the Author}

Olen Gunnlaugson, Ph.D. is an Assistant Professor in leadership and organizational development within the Department of Management at Université Laval, in Quebec City where among other projects, he is co-developing an MBA micro program in Complexity Studies, Consciousness and Leadership with colleagues. Olen brings a multidisciplinary background to leadership studies. His work has been published across peer-reviewed academic journals and presented at numerous international conferences. His main research interests focus on dialogue and collective intelligence approaches to leadership, integral theory, transformative learning processes in groups and teams and contemplative approaches to inquiry.

(C) Copyright 2011. The author, OLEN GUNNLAUGSON, assign to the University of Alberta and other educational and non-profit institutions a non-exclusive license to use this document for personal use and in courses of instruction provided that the article is used in full and this copyright statement is reproduced. The author also grants a non-exclusive license to the University of Alberta to publish this document in full on the World Wide Web, and for the document to be published on mirrors on the World Wide Web. Any other usage is prohibited without the express permission of the authors. 